\title{
Autopercepção de saúde bucal de gestantes e puérperas comparadas aos índices CPOD e CPI
}

Self-perception of oral health of pregnant women and puerperal women compared to CPOD and CPI indices

Wanêssa Trigueiro Casimiro1; Kauanne Fonseca de Lima1; luri Adônis de Souza Nascimento²; Amanda Azevedo Ghersel ${ }^{3}$; Herbert Ghersel ${ }^{4}$; Eloisa Lorenzo de Azevedo Ghersel ${ }^{4}$.

${ }^{1}$ Graduadas de Odontologia da Universidade Federal da Paraíba, João Pessoa - PB - Brasil ${ }^{2}$ Acadêmico de Medicina da Universidade Federal da Paraíba, João Pessoa- PB - Brasil ${ }^{3}$ Acadêmica de Medicina da Faculdade Ciências Médicas da Paraíba, João Pessoa- PB - Brasil ${ }^{4}$ Professores de Odontologia da Universidade Federal da Paraíba, João Pessoa-PB - Brasil

Autor e endereço para correspondência:

Wanêssa Trigueiro Casimiro - Avenida Monteiro da Franca 1383, apt. 204, Manaíra, CEP:58038323. Email: wanessa_casimiro12@hotmail.com

\section{Resumo}

Introdução: O estado de saúde de um indivíduo deve ser visto como um todo, por isso a assistência pré-natal integral auxilia a mulher na redução dos riscos no período gestacional, devendo a saúde bucal também fazer parte desta integralidade. Objetivo: investigar a autopercepção de saúde bucal e compará-la a exames clínicos, além de investigar alguns conceitos básicos referentes à saúde bucal de gestantes e puérperas. Metodologia: A amostra foi constituída por 77 gestantes e puérperas. Realizou-se entrevista subjetiva seguida do exame bucal para obtenção dos índices CPOD e CPI para fins comparativos. Os dados foram analisados e foram considerados os valores relativos e absolutos. Resultados: Quanto à autopercepção da sua saúde bucal, 71,43\% $(n=55)$ achavam que tinham lesões de cárie, 57,14\% $(n=44)$ percebiam sangramento gengival e $77,92 \%(n=60)$ acreditavam que precisavam fazer tratamento dentário. $O$ CPOD médio foi de 9,03 e o CPI 7,03. Quando comparadas a autopercepção aos resultados dos índices, a maioria demonstrou respostas compatíveis, porém algumas tiveram percepções distorcidas. Considerando conceitos básicos sobre saúde bucal, quando perguntadas se problemas dentários poderiam afetar a gravidez, 31,17\% ( $n=24)$ responderam que não, ao passo que $16,88 \%(n=13)$ creem que grávidas não podem fazer tratamento dentário; $58,44 \%(n=45)$ desconhecem as causas das doenças cárie e periodontal. Conclusão: A autopercepção da saúde bucal foi condizente com as avaliações clínicas. A maioria apresenta relativo conhecimento sobre conceitos básicos de saúde bucal, porém em função da alta prevalência das doenças cárie e periodontal sugere-se maior assistência a esta população.

Descritores: Cárie dentária. Cuidado pré-natal. Saúde bucal. 


\begin{abstract}
Introduction: The health status of an individual should be seen as a whole, so integral prenatal care assists women in reducing risks in the gestational period, and oral health should also be part of this integrality. Objective: to investigate the self-perception of oral health and to compare it with clinical exams, in addition to investigate some basic concepts regarding the oral health of pregnant women and puerperal women. Methodology: The sample consisted of 77 pregnant women and postpartum women. A subjective interview was carried out followed by oral examination to obtain the CPOD and $\mathrm{CPI}$ indexes for comparative purposes. The data were analyzed and the relative and absolute values were considered. Results: Regarding the self-perception of their oral health, $71.43 \%(n=55)$ believed they had carious lesions, $57.14 \%(n=44)$ perceived gingival bleeding and $77.92 \%(n=60)$ needed dental treatment. The mean CPOD was 9.03 and the CPI 7.03. When comparing self-perception with index results, most showed compatible responses, but some had distorted perceptions. When considering whether dental problems could affect pregnancy, $31.17 \%(n=24)$ answered that no, while $16.88 \%$ $(n=13)$ believe that pregnant women could not do dental treatment ; 58.44\% $(n=45)$ are unaware of the causes of caries and periodontal diseases. Conclusion: Self-perception of oral health was consistent with clinical evaluations. Most of them present a relative knowledge about basic concepts of oral health, but due to the high prevalence of caries and periodontal diseases, it is suggested that this population be more assisted.
\end{abstract}

Keywords: Dental caries. Prenatal care. Oral health.

\title{
Introdução
}

A Odontologia evoluiu de forma considerável nos últimos anos, o conhecimento efetivo da etiologia mudou a abordagem das doenças cárie e periodontal possibilitando prevenir, reverter e trata-las. No passado, estas afecções foram a causa de perdas dentárias em crianças e adultos, e apesar da melhoria expressiva das condições de saúde bucal na população mundial ${ }^{1}$, ainda hoje estão concentradas em uma população vulnerável com menor poder socioeconômico e cultural, susceptível a uma série de enfermidades. Portanto, o principal cerne da promoção de saúde e da prevenção deve voltar-se a estes indivíduos que consomem grande parte dos recursos destinados ao controle e tratamento de doenças, além de disseminá-las. Mães são importantes fontes de transmissão de microrganismos bucais patogênicos para seus filhos, assim o trabalho educativo com mães e gestantes previne doenças nas futuras gerações².

Em muitos casos, a cárie dentária e a doença periodontal possuem evolução clínica lenta e assintomática, fazendo com que o indivíduo busque cuidados em estágios mais avançados, principalmente, quando a dor se associa. $O$ indivíduo deve ser esclarecido e orientado sobre 0 
desenvolvimento para que possa evita-las ou reconhece-las desde os processos iniciais, assim a prevenção se torna efetiva.

O Hospital Universitário Lauro Wanderley da Universidade Federal da Paraíba (HULW UFPB) e o Instituto Cândida Vargas, locais de referência em atendimento de gestantes de alto risco na cidade de João Pessoa/PB, prestam serviços de pré-natal, obstetrícia e puericultura a mulheres que, em sua maioria, pertencem a classes sociais mais desfavorecidas e que precisam de cuidados não só assistenciais, mas também de prevenção e educação em saúde.

Partindo-se da hipótese que gestantes e puérperas atendidas nestes hospitais enquadram-se no perfil supracitado, considerando que o conhecimento das necessidades de tal população possa contribuir para a implementação de medidas assistenciais e socio educativas que fortaleçam a saúde da gestante e do bebê, é que esta pesquisa se insere.

Portanto, o objetivo deste estudo foi investigar a autopercepção de saúde bucal e compará-la a exames clínicos, além de investigar alguns conceitos básicos referentes à saúde bucal de gestantes e puérperas.

\section{Metodologia}

Este é um estudo descritivo, com abordagem quantitativa. A amostra foi composta por 16 gestantes atendidas no ambulatório de pré-natal do Hospital Universitário Lauro Wanderley-UFPB e 61 puérperas que ainda não haviam recebido alta das enfermarias do Instituto Cândida Vargas, totalizando 77 mulheres. O cálculo da amostra baseou-se na população estimada atendida nessas instituições no intervalo de tempo do estudo que durou quatro meses. Foram incluídas no estudo 16 mulheres em variados períodos gestacionais e 61 puérperas com idade gestacional que variou entre 26 e 41 semanas. Foram excluídas mulheres que se negaram a participar da pesquisa e/ou exame bucal.

A pesquisa foi submetida à aprovação ao Comitê de Ética em Pesquisa do Hospital Universitário Lauro Wanderley, por meio da Plataforma Brasil e foi aprovado sob o parecer n: 1.626.106. Aplicou-se o Termo de Consentimento Livre e Esclarecido (TCLE), realizou-se entrevistas com perguntas fechadas direcionadas e, a seguir, foram realizados exames bucais para avaliação do Índice cárie (COPD - Dentes Cariados, Perdidos e Obturados) e periodontal (CPI Índice Periodontal Comunitário). Logo após, os prontuários das respectivas participantes também foram examinados para confirmação de alguns dados. 
Conforme Pinto $^{3}$, em levantamentos mais simples cujos objetivos se limitam ao conhecimento de uma determinada situação, como é o caso do presente estudo, a padronização de critérios de exame é suficiente para reduzir a ocorrência de erros intra e inter-examinadores, portanto esta medolologia foi aplicada aos discentes do curso de Odontologia, pelos professores orientadores. Os exames das gestantes foram realizados nos consultórios odontológicos da disciplina de Clínica Integrada, da Faculdade de Odontologia da UFPB e das puérperas foram feitos no leito das enfermarias do Instituto Cândida Vargas. Os instrumentais, materiais descartáveis e de proteção individuais foram fornecidos pela própria equipe de pesquisadores.

De acordo com o índice CPOD, a experiência de cárie foi considerada como ausente (CPOD igual a zero); baixa (valores de CPO-D entre 1 e 4); moderada (valores de CPO-D entre 5 e 8); e alta (valores de CPOD igual ou superior a 9). Para o índice CPI os valores foram considerados por sextante: 0 - sextante hígido; 1 - sextante com sangramento; 2 - cálculo; 3 - bolsa de $4 \mathrm{~mm}$ a $5 \mathrm{~mm}$; 4 - bolsa de $6 \mathrm{~mm}$ ou mais; $X$ - sextante excluído (menos de 2 dentes presentes); 9 - sextante não examinado 4 .

Os dados foram analisados utilizando-se planilha Excel ${ }^{\circledR}$ e o software estatístico SPSS ${ }^{\circledR}$, versão 20.0, considerando valores relativos e absolutos.

\section{Resultados}

Do total de 77 (100,00\%) participantes do estudo, 16 (20,78\%) eram gestantes, enquanto $61(79,22 \%)$ eram puérperas.

Quando questionadas em relação à percepção da sua saúde bucal, $71,43 \% \quad(n=55)$ achavam que tinham lesões de cárie e 57,14\% ( $n=44)$ disseram que suas gengivas sangravam. Além disso, $77,92 \%(n=60)$ acreditavam que estavam precisando fazer tratamento dentário (Tabela 1).

\begin{tabular}{cccc}
\hline Tabela 1 - Percepção da própria saúde bucal e frequências & & \\
\hline & & Frequência & Percentual \\
Acha que tem cárie dentária? & Não & 22 & 28,57 \\
& Sim & 55 & 71,43 \\
Gengivas sangram? & Não & 33 & 42,86 \\
& Sim & 44 & 57,14 \\
Está precisando de tratamento dentário? & Não & 17 & 22,08 \\
& Sim & 60 & 77,92
\end{tabular}


Quando examinadas clinicamente, o índice CPOD variou de 0,00 a 22,00, com média de 9,03 ( $s d=4,87$ ), enquanto o CPI variou entre um mínimo de 0,00 e um máximo de 18,00, com média de 7,03 e sd =3,91 (Tabela 2).

\begin{tabular}{cccccc}
\hline \multicolumn{2}{l}{ Tabela 2 - Índices CPOD e CPI } & & & & \\
\hline Variável & $\mathbf{N}$ & Média & Desvio padrão & Mínimo & Máximo \\
CPOD & 77 & 9,03 & 4,87 & 0,00 & 22,00 \\
CPI & 77 & 7,03 & 3,91 & 0,00 & 18,00
\end{tabular}

A tabela 3, mostra os valores acima de zero do índice CPOD separados em dentes cariados, perdidos e obturados. A média de dentes cariados na amostra foi de 3,64, variando de 1,00 a 17,00 .

\begin{tabular}{ccccccc}
\hline \multicolumn{2}{l}{ Tabela 3 - Componentes C, P e O* } \\
\hline & N & Percentual & Mínimo & Máximo & Média & Desvio Padrão \\
CPOD_C & 61 & 79,2 & 1,00 & 17,00 & 3,64 & 3,00 \\
CPOD_P & 53 & 68,8 & 1,00 & 12,00 & 3,89 & 3,19 \\
CPOD_O & 53 & 68,8 & 1,00 & 13,00 & 5,02 & 3,14
\end{tabular}

*Apenas pacientes com valores $>0$

$\mathrm{Na}$ tabela 4, comparou-se os valores de dentes cariados obtidos pelo índice CPOD com a autopercepção de cárie dentária. Os números indicam uma percepção equivocada da saúde bucal de alguns sujeitos da pesquisa, pois 5 mulheres que não tinham lesões cariosas afirmaram ter. Por outro lado, 11 acreditavam não ter lesões de cárie, mas tinham.

\begin{tabular}{|c|c|c|c|c|}
\hline & & \multicolumn{2}{|c|}{ Acha que tem cáries? } & \multirow{2}{*}{ Total } \\
\hline & & Não & Sim & \\
\hline \multirow{12}{*}{ CPOD_C } & ,00 & 11 & 5 & 16 \\
\hline & 1,00 & 5 & 10 & 15 \\
\hline & 2,00 & 2 & 11 & 13 \\
\hline & 3,00 & 2 & 9 & 11 \\
\hline & 4,00 & 0 & 4 & 4 \\
\hline & 5,00 & 1 & 5 & 6 \\
\hline & 6,00 & 0 & 3 & 3 \\
\hline & 7,00 & 1 & 2 & 3 \\
\hline & 8,00 & 0 & 1 & 1 \\
\hline & 9,00 & 0 & 2 & 2 \\
\hline & 10,00 & 0 & 2 & 2 \\
\hline & 17,00 & 0 & 1 & 1 \\
\hline Total & & 22 & 55 & 77 \\
\hline
\end{tabular}

Quando abordada a questão periodontal, a tabela 5 relaciona a percepção de sangramento gengival aos valores do exame bucal através do índice CPI 


\begin{tabular}{|c|c|c|c|c|}
\hline & & \multicolumn{3}{|c|}{ Suas gengivas sangram? } \\
\hline & & Não & Total & \\
\hline & ,00 & 2 & 1 & 3 \\
\hline & 1,00 & 2 & 2 & 4 \\
\hline & 2,00 & 4 & 1 & 5 \\
\hline & 3,00 & 1 & 1 & 2 \\
\hline & 4,00 & 2 & 3 & 5 \\
\hline & 5,00 & 2 & 3 & 5 \\
\hline & 6,00 & 6 & 9 & 15 \\
\hline & 7,00 & 2 & 3 & 5 \\
\hline CPI & 8,00 & 3 & 4 & 7 \\
\hline & 9,00 & 3 & 4 & 7 \\
\hline & 10,00 & 2 & 2 & 4 \\
\hline & 11,00 & 2 & 2 & 4 \\
\hline & 12,00 & 0 & 3 & 3 \\
\hline & 13,00 & 0 & 4 & 4 \\
\hline & 14,00 & 2 & 0 & 2 \\
\hline & 15,00 & 0 & 1 & 1 \\
\hline & 18,00 & 0 & 1 & 1 \\
\hline Total & & 33 & 44 & 77 \\
\hline
\end{tabular}

Em relação aos conceitos sobre saúde bucal durante a gestação, quando perguntadas se achavam que problemas dentários poderiam afetar a gravidez, 24 (31,17\%) responderam que não, ao passo que $13(16,88 \%)$ creem que grávidas não podem ser submetidas a tratamento dentário e $45(58,4 \%)$ desconhecem as causas das doenças cárie e periodontal.

\section{Discussão}

O estado de saúde de um indivíduo deve ser visto como um todo, por isso a assistência pré-natal integral auxilia a mulher na redução dos riscos no período gestacional, devendo a saúde bucal também fazer parte desta integralidade.

A saúde bucal no Brasil depara-se com imenso paradoxo, a Odontologia é considerada de vanguarda em relação aos padrões mundiais, concomitantemente, a saúde bucal de grande parte da população é precária pois as condições socioeconômicas e oferta de serviços limitam o acesso ${ }^{5}$. Em se tratando de gestantes, o quadro pode agravar-se porque, somado às condições econômicas e falta de acesso aos serviços, ainda existem barreiras culturais ligadas a medos e mitos a respeito de tratamento buco dentário no período gestacional. Além disso, durante a gestação, mudanças de hábitos, principalmente alimentares e de higiene, podem gerar alterações no equilíbrio do 
ambiente bucal e favorecer a instalação das doenças cárie e periodontal ou podem agravar as doenças pré-existentes ${ }^{6}$.

$\mathrm{Na}$ presente investigação, fez-se, através de entrevistas, uma análise subjetiva considerando a autopercepção dos sujeitos frente à presença ou não de cárie dentária e sangramento gengival, à interpretação das doenças bucais e ao tratamento dentário durante a gestação. Em revisão sistemática sobre autopercepção de saúde bucal e fatores associados, autores $^{8}$ concluíram que avaliações subjetivas devem ser consideradas suportes críticos para indicadores clínicos que podem auxiliar na identificação de pessoas ou grupos populacionais em situação de vulnerabilidade que necessitam de cuidados assistenciais. A percepção de saúde oral está fortemente associada às condições biológicas, individuais e à contextualização das determinantes sociais, quanto pior a condição menor a percepção da pessoa em diferenciar o grau de saúde e doença ${ }^{7}$.

A maioria das pacientes atendidas nos hospitais referenciados neste estudo tem como características gerais condições sociais, econômicas e culturais desfavoráveis, porém apesar destas particularidades, diferente do que se poderia esperar ${ }^{8}$, quando questionadas em relação à percepção da sua saúde bucal, mais da metade $71,43 \%(n=55)$ conseguiu perceber que tinha lesões de cárie e 57,14\% ( $n=44)$ relataram sangramento gengival. Além disso, 60 (77,92\%) percebiam a necessidade de tratamento dentário (Tabela 1), mas não trataram durante a gestação nem estavam em tratamento, o que condiz com estudos que relatam baixa procura pelo cirurgião dentista no período gestacional ${ }^{9}$.

No presente estudo, os valores do índice CPOD variaram de 0,00 a 22,00, com média de 9,03 (sd $=4,87$ ) (Tabela 2 ), sendo que 61 sujeitos da amostra $(79,2 \%)$ apresentavam dentes cariados, $53(68,8 \%)$ perdidos e $53(68,8 \%)$ obturados (Tabela 3). Estes valores representam alta experiência da doença cárie nesta população, padrões semelhantes têm sido reportados na literatura, o que gera preocupação por se tratar de mulheres em fase reprodutiva ${ }^{10,11}$.

Apesar da maioria $(71,43 \%, n=55)$ ter achado que tinha lesões de cárie, quando se comparou a autopercepção ao exame clínico profissional observou-se uma certa proporcionalidade, porém, houveram controvérsias na percepção da doença, pois cinco participantes achavam que tinham lesões de cárie e ao exame clínico não se constatou a presença de tais lesões. Da mesma forma, 11 mulheres afirmaram que não tinham cárie dentária e após o exame clínico foi constatada a presença de lesões (Tabela 4).

Em relação aos conceitos sobre saúde bucal durante a gestação, quando interpeladas se achavam que problemas dentários poderiam afligir a gravidez, $24(31,17 \%)$ responderam que não. 
A desinformação fez com que 13 delas (16,88\%) acreditassem que grávidas não podem ser submetidas a tratamento odontológico, além disso 45 (58,4\%) desconhecem as causas das doenças cárie e periodontal. Esta falta de informação ainda presente numa população responsável não só pela sua saúde, mas pela de seus filhos e do núcleo familiar, contribui para a precariedade da saúde bucal e pelo distanciamento das mulheres da atenção odontológica ${ }^{12}$.

O levantamento nacional em 2003, que avaliou a condição periodontal brasileira através do Índice Periodontal Comunitário (CPI), apontou de doença periodontal em 53,5\% da amostra entre 15 a 19 anos e 67,7\% entre 35 a 44 anos de idade, pode-se observar que a doença se agrava com o avanço da idade ${ }^{11,13}$. No presente estudo, a maioria $57,14 \%(n=44)$ relatou a percepção de sinais de inflamação e sangramento gengival, o que foi confirmado através dos valores CPI cuja média foi de 7,03 , valores altos do índice CPI sugerem alta prevalência da doença, a literatura apresenta expressões similares ${ }^{8,14}$.

Nessa questão também houve fatores inquietantes, pois 11 mulheres com índices de CPI acima de 10 afirmaram não perceber o sangramento gengival - que é um dos primeiros sinais de inflamação gengival. Além disso, 1 sujeito relatou sangramento e ao exame bucal não apresentava sinais de doença. As divergências entre a autopercepção e os exames bucais provavelmente se justificam pela dificuldade que alguns indivíduos tem em se perceber ${ }^{8}$.

\section{Conclusão}

A maioria das mulheres da amostra estudada consegue autoperceber a presença de lesões cariosas e sangramento gengival.

Quando comparada autopercepção ao exame clínico constatou-se compatibilidade entre a maioria das respostas e os valores da avaliação clínica, porém algumas mostravam percepções equivocadas.

A maioria tem um relativo esclarecimento sobre saúde bucal durante a gestação, porém desconhecem as causas das doenças cárie e periodontal.

Diante dos resultados, em razão da alta prevalência das doenças bucais encontrada nesta população sugere-se que medidas assistenciais, ações profiláticas específicas e educação em saúde bucal sejam implementadas. 


\section{Referências}

1. Glass RL, Glass R, Glass R, Glass R, Glass R. The First International Conference on the Declining Prevalence of Dental Caries. J Dent Res. 1982 Jan 1;61(Spec. Iss.):1301-83.

2. Weyne SC. A Construção do Paradigma de Promoção de Saúde - Um desafio para novas gerações. In: ABOPREV - Promoção de saúde bucal. São Paulo: Artes Médicas; 2003. p. $1-24$.

3. Pinto V. Saúde Bucal Coletiva. 6th ed. São Paulo: Editora Santos; 2013.

4. Brasil. Projeto SB2000 - Condições de Saúde Bucal da População Brasileira no ano 2000 Manual do Examinador. Brasília; 2001.

5. Porto CLA, Pereira JC, Anauate Netto C. Cariologia. São paulo: Artes Medicas; 2008. 240 p.

6. Cabral MCB, Santos TS, Moreira TP. Percepção das gestantes do Programa de Saúde da Família em relação à saúde bucal no município de Ribeirópolis, Sergipe, Brasil. Rev Port Saúde Pública. 2013 Jul 1;31(2):160-7.

7. Silva JV, Roncalli AG. Individual and contextual factors associated to the self-perception of oral health in Brazilian adults. Rev Saude Publica. 2018;52:29.

8. Mattos BNC, Davoglio RS. Saúde bucal: a voz da gestante. Rev da Fac Odontol - UPF. 2015; 20(3):393-399 .

9. Scavuzzi AIF, Rocha MCBS, Vianna MIP. Estudo da prevalência da cárie dentária em gestantes brasileiras, residentes em Salvador-BA. JBP, j bras odontopediatr odontol bebê. 1999;2(6):96-102.

10. Martins DP, Borges AH, Segundo AS, Palma VC, Volpato LER. A Saúde Bucal de uma Subpopulação de Gestantes Usuárias do Sistema Único de Saúde: um Estudo Piloto. Pesqui Bras Odontopediatria Clin Integr. 2013;13(3):273-278.

11. Brasil. Projeto SB Brasil 2003: condições de saúde bucal da população brasileira 2002-2003: resultados principais. Ministério da Saúde - Departamento de Atenção Básica; 2004. 51 p.

12. Velasco FG, Mingotti AC, Sallum AW, Passos SD. Condição periodontal em puérperas atendidas em Hospital Universitário da FMJ, Brasil. ImplantNewsPerio. 2017;2(5):912-916.

13. Offenbacher S, Beck JD, Lieff S, Slade G. Role of periodontitis in systemic health: spontaneous preterm birth. J Dent Educ. 1998 Oct;62(10):852-858.

14. Shanthi V, Vanka A, Bhambal A, Saxena V, Saxena S, Kumar SS. Association of pregnant women periodontal status to preterm and low-birth weight babies: A systematic and evidencebased review. Dent Res J (Isfahan). 2012 Jul;9(4):368-380. 